\title{
Preparing Modified Hyaluronic Acid with Tunable Content of Vinyl Groups for Use in Fabrication of Scaffolds by Photoinduced Crosslinking
}

\author{
A. V. Sochilina ${ }^{a, b, 1}$, A. G. Savelyev ${ }^{b, c}$, R. A. Akasov ${ }^{a, b}, c$, V. P. Zubov ${ }^{a}$, \\ E. V. Khaydukov ${ }^{a, b, c}$, and A. N. Generalova ${ }^{a}$ \\ ${ }^{a}$ Shemyakin-Ovchinnikov Institute of Bioorganic Chemistry, Russian Academy of Sciences, Moscow, 117997 Russia \\ ${ }^{b}$ Federal Scientific Research Centre "Crystallography and Photonics”, Russian Academy of Sciences, Moscow, 119333 Russia \\ ${ }^{c}$ Sechenov First Moscow State Medical University, Moscow, 119991 Russia \\ Received August 17, 2020; revised August 20, 2020; accepted August 26, 2020
}

\begin{abstract}
Materials based on hyaluronic acid (HA) are extensively used in tissue engineering as scaffolds. Photoinduced crosslinking is one way to prepare them, and, for this, HA must be modified with vinyl groups, which are capable of participating in free-radical reactions upon exposure to light. The quantity of grafted vinyl groups, represented as the degree of substitution (DS), is an important parameter of modified HA (mHA) that is related to the mechanical, chemical, and biological properties of scaffolds. Here, we demonstrate the feasibility of tuning DS by varying the reaction parameters (composition and concentration of reaction components and reaction conditions) and investigate the effect of DS on the viscosity of mHA solutions. As example, we consider the photoinduced reaction of $\mathrm{mHA}$ in the presence of flavin mononucleotide as the initiator, which can be used in fabrication of noncytotoxic scaffolds by $3 \mathrm{D}$ printing. The growth behavior of fibroblasts on the scaffold surface is studied.
\end{abstract}

Keywords: hyaluronic acid, glycidyl methacrylate, scaffolds, polymer analogous reactions

DOI: $10.1134 / \mathrm{S} 1068162021040191$

\section{INTRODUCTION}

Tissue engineering, an innovative research area aimed at creating constructs that can support, regenerate, and replace damaged tissues and organs, is currently under intensive development. These constructs are based on scaffolds, which are three-dimensional frameworks which cells can attach to and proliferate on their surface and inside [1-3]. After a scaffold is implanted at a lesion site, new biological tissue starts growing there, and the initial framework degrades or is removed surgically. Scaffolds for tissue engineering must meet a number of requirements [4, 5]: 1) biocompatibility and absence of cytotoxicity, immunogenicity, and pyrogenicity of the construct itself as well as its degradation products; 2) amenability to biodegradation, which enables replacement of artificial constructs with body's own tissues; 3) surface properties that facilitate adhesion and proliferation of cells and preservation of their function; 4) mechanical integrity

Abbreviations: HA, hyaluronic acid; mHA, modified hyaluronic acid; GMA, glycidyl methacrylate; DMF, dimethylformamide; MTT, 3-(4,5-dimethylthiazol-2-yl)-2,5-diphenyltetrazolium bromide; PEGDA, poly(ethylene glycol) diacrylate; DS, degree of substitution; TEA, triethylamine; TEAB, tetraethylammonium bromide; and PCC, photocurable composition.

${ }^{1}$ Corresponding author: e-mail: ddraig@yandex.ru. similar to that of the tissue being replaced; 5) porosity optimal for unhampered distribution of cells and extracellular matrix that they produce, as well as for efficient metabolism; and at the same time the mechanical integrity and porosity must be in balance.

In addition to the requirements listed above, a method for scaffold fabrication must be reproducible, scalable, and commercially feasible. Scaffolds can be fabricated on the basis of metals, ceramics, synthetic and natural polymers, or their composites. Metal- and ceramic-based scaffolds are not amenable to degradation, which may result in complications in the long term. Using synthetic polymers that have a predominantly hydrophobic surface in fabrication of scaffolds hinders cell attachment and contributes to the formation of toxic degradation products, as is the case, e.g., with an FDA-approved poly(lactide-co-glycolide) copolymers [4-6]. Recently, materials based on natural polymers and their derivatives have attracted considerable attention; this especially concerns endogenic compounds, because they a priori exhibit good biocompatibility and propensity to enzymatic degradation in the body. Hyaluronic acid (HA), a linear polysaccharide consisting of unbranched repeat units of glucuronic acid and $N$-acetylglucosamine, is one such polymer. As a major component of an extracellular 
matrix, HA is found in connective, epithelial, and nerve tissues, and in many biological fluids (e.g., saliva and synovial fluid); it actively participates in proliferation and migration of cells $[7,8]$. However, preparation of HA-based scaffolds is hindered because of its extremely high swelling coefficient and the inability of polysaccharide in the hydrogel form to retain the required shape.

Methods for preparing composite HA-based scaffolds have been developed. In these scaffolds, HA is linked via noncovalent bonds, e.g., to collagen, silk fibroin, complementary DNA, or polyvinyl alcohol [9-12]. In addition, bonding is created by modifying HA with thermoresponsive polymers [13]. Noncovalent crosslinking does not resolve the issue of inadequate mechanical integrity of HA hydrogels, and it is therefore advantageous to develop methods for fabrication of HA-based scaffolding materials by reactions leading to intramolecular and intermolecular crosslinking of HA chains. One approach is to modify HA by grafting vinyl groups and performing free-radical crosslinking, which can be implemented not only by using chemical reagents, but also by exposure to light. Photoinduced crosslinking reactions enable us to create materials with tailored physicochemical properties while preserving their biocompatibility and ability for biodegradation. Scaffolds can be fabricated by micromolding or 3D laser printing [14-17]; in addition, a construct can be prepared directly in a living body, i.e., without the preliminary in vitro stage [18].

Polymer-analogous reactions involving methacrylic anhydride or glycidyl methacrylate (GMA) are widely used in preparing HA derivatives containing vinyl groups [19-22]. The concentration of grafted vinyl groups, which can be expressed as the degree of substitution (DS) of polymer's groups with GMA residues, is the core parameter used in preparing such derivatives. DS influences not only the degree of crosslinking but also cytotoxicity, amenability to biodegradation, and the mechanical qualities of prepared scaffolds. Controlling DS is the key to preparation of scaffolds with tailored properties. DS can be estimated by IR [23] or ${ }^{1}$ H NMR [20, 22] spectroscopies; however, the precise determination of DS by these methods is hampered due to high hygroscopicity and the polymeric nature of modified HA (mHA), which contributes to peak broadening and the presence of much noise. Here, we determined DS quanitatively by a titrimetric method involving potassium permanganate, which was developed earlier [24]. DS was used as the parameter that enables comparison between different mHA samples and, accordingly, identifying the effects of reaction conditions on the progress of polymeranalogous reactions.

The aim of this work is to investigate the effect that the parameters of reaction between HA and GMA (reaction conditions and composition of reaction mixture) have on DS, inasmuch as DS determines the properties of mHA and scaffolds based on it. In this work, we developed a technique for preparation of scaffolds that relies on a photocuring reaction in which flavin mononucleotide, an endogenous and nontoxic compound, is used as the initiator. We investigated cytotoxic qualities of prepared scaffolds and the potential for cultivating cells on their surface.

\section{RESULTS AND DISCUSSION}

A polymer-analogous reaction between $\mathrm{HA}$ and GMA to graft units with a double bond can be implemented as either a heterogeneous reaction in an aqueous medium containing GMA, which is poorly soluble in water [19], or a homogeneous reaction in organic solvents after the substitution of the lipophilic tetrabutyl ammonium ion for sodium ions in HA [20], or a homogeneous reaction in a mixed aqueous-organic medium [21]. In this work, to modify HA by using GMA, we chose a facile and easy-to-control reaction in a mixed aqueous-organic medium [21]. As a result of carrying out this reaction in a water-DMF mixed solvent (ratio, $1.6: 1)(0.36 \mathrm{wt} \%$ of HA, $0.36 \mathrm{wt} \%$ of tetraethylammonium bromide (TEAB) and $18 \mathrm{~mL}$ of GMA per $1 \mathrm{~g}$ of $\mathrm{HA}$ ) at $25^{\circ} \mathrm{C}$ for four days, we obtained samples in which the substitution of units containing double bonds for HA groups, i.e., DS, constituted $40 \%$. To analyze the effect that parameters of the reaction between HA and GMA have on DS, which is needed to control DS, we varied $\mathrm{pH}$, temperature, duration of reaction, and the HA/GMA ratio in the reaction mixture and studied the contribution of a phase transfer catalyst.

\section{Effect of $p H$ on the DS of $m H A$}

The effect of $\mathrm{pH}$ on the DS of mHA was evaluated for the initial reaction mixture at a neutral $\mathrm{pH}(6-7)$, in an alkaline medium $(\mathrm{pH} 12)$ that resulted from substitution of tetraethylammonium bromide (TEAB) for the triethylamine (TEA) catalyst, and in acidic medium ( $\mathrm{pH} 4.5$ ) that was created by adding $\mathrm{HCl}$ to the initial reaction mixture. We found that the DS of samples prepared in the alkaline medium was half that $(21 \%)$ of samples prepared in acidic or neutral media ( $40-41 \%)$, when all other factors were held constant. According to literature [21], the polymer-analogous reaction between HA and GMA in a mixed aqueousorganic medium proceeds via two main pathways: reversible transesterification of methacrylic acid moiety of the GMA molecule and irreversible reaction with opening of the glycidyl's epoxide ring (Fig. 1). Using model polymers containing only hydroxyl functionalities (polyvinyl alcohol) or only carboxyl functionalities (polyacrylic acid) as examples, Reis et al. corroborated these mechanisms [25]. It was shown that at $\mathrm{pH} 3.5$ GMA reacted with both hydroxyl and carboxyl groups via epoxide ring opening, and at $\mathrm{pH} 10.5 \mathrm{GMA}$ underwent hydrolysis and reacted only with hydroxyl 


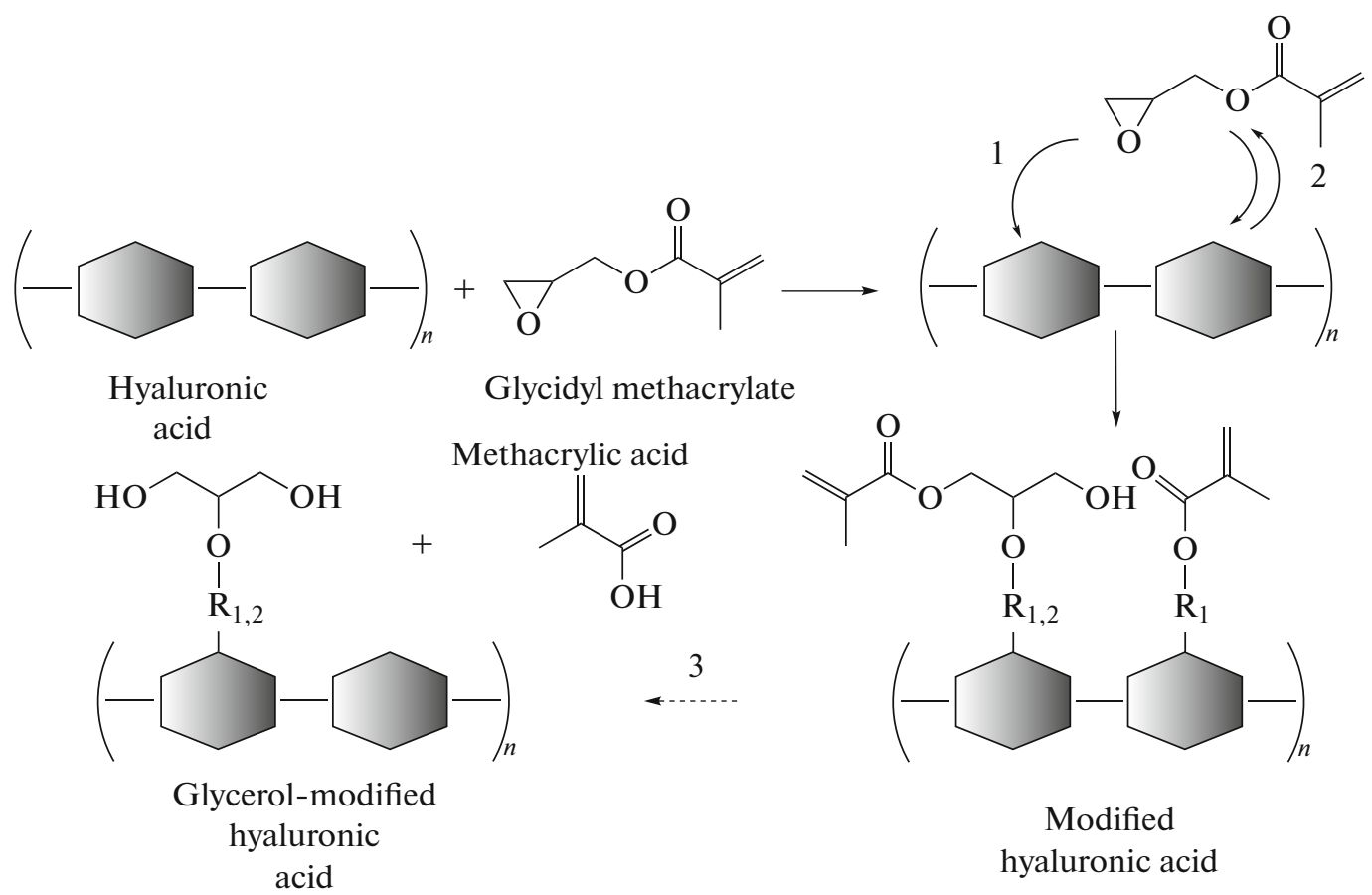

Fig. 1. Reaction schematics for modification of hyaluronic acid with glycidyl methacrylate: $(1)$ irreversible reaction of epoxide ring opening, (2) reversible transesterification reaction, and (3) hydrolysis. $\mathrm{R}_{1}=\mathrm{CH}_{2}, \mathrm{R}_{2}=\mathrm{C}=\mathrm{O}$.

groups via the two pathways, with the reaction predominantly proceeding via the epoxide ring-opening pathway. A complex mechanism of reaction between polysaccharides and GMA was addressed by Li et al. [26]. More specifically, the reaction between chondroitin sulfate and GMA was established to give rise to different reaction products under alkaline $(\mathrm{pH} 8.5)$ and close-to-neutral $(\mathrm{pH}$ 6.4) conditions, which is due to several concomitant processes: initially, transesterification and modification via opening of the epoxide ring occurred, and the resulting products can then undergo hydrolysis to form the unmodified polysaccharide and the polysaccharide containing glycerol, methacrylic acid, and glycidyl moieties. We note that the hydrolysis and transesterification reaction

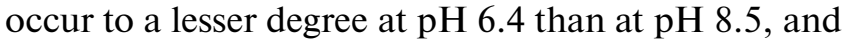
at $\mathrm{pH} 3.0$ the hydrolysis of ester bonds is nearly absent; therefore, the modification largely proceeds via opening of the epoxide group.

Due to the mechanisms described above, we can conclude that at alkaline $\mathrm{pH}$ the hydrolysis occurs alongside grafting of vinyl groups, which reduces the DS of the final product. In addition, very similar values for DS ( 40-41\%) observed under neutral and acidic conditions suggest that hydrolysis is insignificant at neutral $\mathrm{pH}$. As a result, the optimal process is achieved when the reaction HA modification is carried out in a neutral medium in which DS can be adjusted by reducing the contribution of side reaction to a minimum. TEAB is the most appropriate catalyst for this process, because it does not raise reaction $\mathrm{pH}$ and functions as a phase transfer catalyst, which enables us to carry out the reaction in both aqueous and organic media.

\section{The Effects of Duration and Temperature of Reaction on the DS of $\mathrm{mHA}$}

The reaction of HA modification was carried out for four days, compared with processes described in literature that were carried out at $\mathrm{pH} 12$ for 5-10 days [21]. This protocol was chosen based on the results of our earlier study [24] in which the DS of the reaction product was monitored on a daily basis during a sixday period. We found that after four days, DS was not changing appreciably during further progress of the reaction. We observed good reproducibility in terms of DS, and the desired degree of crosslinking in photoinduced formation of hydrogels was achieved. Performing the reaction at neutral $\mathrm{pH}$ reduced hydrolysis appreciably and, thus, raised DS; so, at $25^{\circ} \mathrm{C}$, the optimal reaction duration was 4 days.

Raising the reaction temperature from 25 to $40^{\circ} \mathrm{C}$ enabled us to shorten the duration of reaction while increasing DS (Fig. 2). For instance, at $25^{\circ} \mathrm{C}$, it took four days to achieve a DS of HA of $40 \%$, but only two days at $40^{\circ} \mathrm{C}$. This may be related to the Van't Hoff law holding true at the higher temperature, i.e., the rate of irreversible reaction of epoxide ring opening increases; in addition, the solubility of GMA in the reaction mixture may increase as well. Raising the temperature fur- 


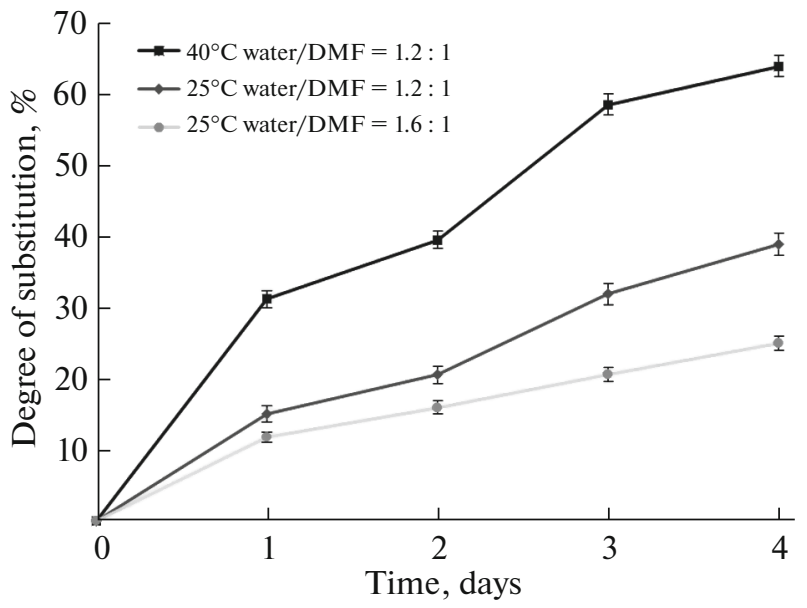

Fig. 2. Dependence of the degree of substitution (DS) of mHA on the duration of reaction with glycidyl methacrylate $(18 \mathrm{~mL}$ per $1 \mathrm{~g}$ of $\mathrm{HA})$ carried out at 25 or $40^{\circ} \mathrm{C}$ at a water/DMF ratio of $1.6: 1$ (with TEAB) and $1.2: 1$ (no $\mathrm{TEAB}$ ). Data shown are the averages of three parallel measurements.

ther may result in thermal hydrolysis of HA and lowering of the molecular weight of the products [27].

\section{The Effect of Reaction Duration and Proportion between the Components on the DS of $\mathrm{mHA}$}

Changing the concentration of reagents in the initial mixture is one effective way to control the DS of $\mathrm{HA}$ in its polymer analogous reactions. Raising the concentration of organic component by increasing the water/DMF ratio from $1.6: 1$ to $1.2: 1$ enabled us to prepare mHA with nearly the same content of vinyl groups $(\sim 40 \%)$ on the fourth day of reaction at $25^{\circ} \mathrm{C}$, while not adding TEAB (Fig. 2). This effect may be related to the increase in DMF content, which improved the GMA solubility and thus contributed to the formation of a more homogeneous system that does not require the use of the phase transfer catalyst TEAB.

DS can be effectively adjusted by varying the GMA concentration in the reaction mixture, when all other factors are held constant. Raising the GMA concentration in the system led to an increase in DS, which can be as high as $64 \%$ (Fig. 3). The experiment was carried out at $40^{\circ} \mathrm{C}$ and a water/DMF phase ratio of $1.2: 1$.

Importantly, increasing the proportion of GMA in excess of $18 \mathrm{~mL}$ of GMA per $1 \mathrm{~g}$ of HA did not lead to a considerable increase in DS within the considered time frame, suggesting that it is unreasonable to raise further the GMA content of the reaction mixture. It is also worth noting that raising the HA concentration from 0.36 to $0.56 \mathrm{wt} \%$, when all other parameters were held constant, did not have a marked effect on the final DS, but it allowed us to lower the consumption of all the other reagents in the reaction mixture.

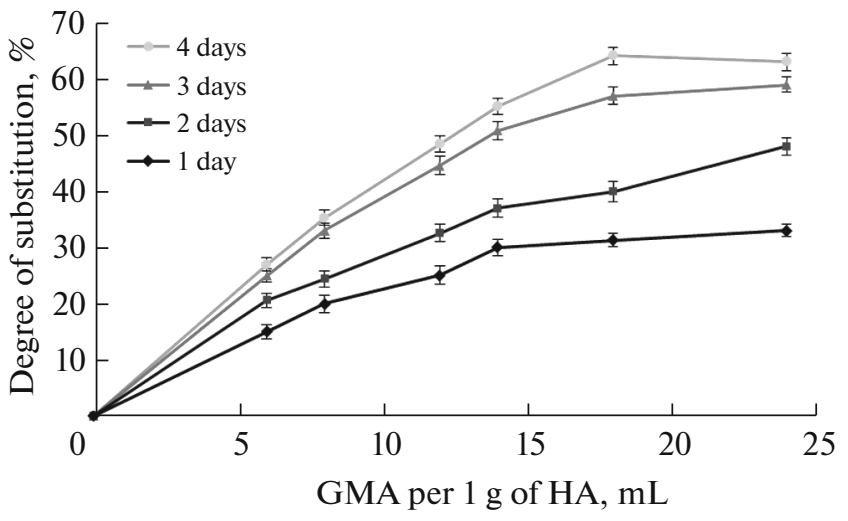

Fig. 3. Variation of the degree of substitution with the proportion of glycidyl methacrylate in the reaction mixture at different reaction durations (all reactions here were carried out at $40^{\circ} \mathrm{C}$ and a water/DMF ratio of $1.2: 1$ ). Data shown are the averages of three parallel measurements.

As a result, to prepare mHA with highest DS, the reaction must be carried out in a neutral medium at elevated temperature $\left(40^{\circ} \mathrm{C}\right)$ for at least four days, with the GMA content being $\geq 18 \mathrm{~mL}$ per $1 \mathrm{~g}$ of $\mathrm{HA}$.

\section{The Effect of DS on the Viscosity of $m H A$ Solutions}

An important descriptor of mHA is the viscosity of its solutions, which has a great impact on the mobility of free radicals in a reaction of photoinduced crosslinking. We measured the kinematic viscosity of diluted solutions $(0.5 \mathrm{wt} \%)$ of mHA with different DS by using capillary viscometry (Fig. 4). Reduction in viscosity that occurs with increasing DS may be related to an increase in the quantity of hydrophobic GMA units. As a results, the number of hydrogen bonds falls, which thus leads to diminishing of hydration shells around HA molecules and a decrease in the intermolecular friction. Despite the fact that the measurements were performed for diluted mHA solutions, this trend, which is related to the decrease in viscosity with increasing DS, was observed for photocurable compositions (PCCs) with a high mHA content ( $20 \mathrm{wt} \%)$ as well, which has a great impact on the parameters of $3 \mathrm{D}$ printing.

\section{mHA-Based Scaffolds}

For extrusive 3D printing, the optimal range of DS of $\mathrm{mHA}$ was established to be $30-55 \%$, which ensures the viscosity required for preparation of scaffolds with desired qualities. For DS $<30 \%$, high viscosity of samples makes extrusion difficult and prevents fabrication of scaffolds with the desired degree of crosslinking. For DS $>55 \%$, it was impossible to prepare a sample that would retain its shape upon extrusion. We chose mHA with a vinyl group content of $40 \%$ as the optimal variant of DS for extrusion; PCCs prepared based on it were used in 3D printing, as described in 


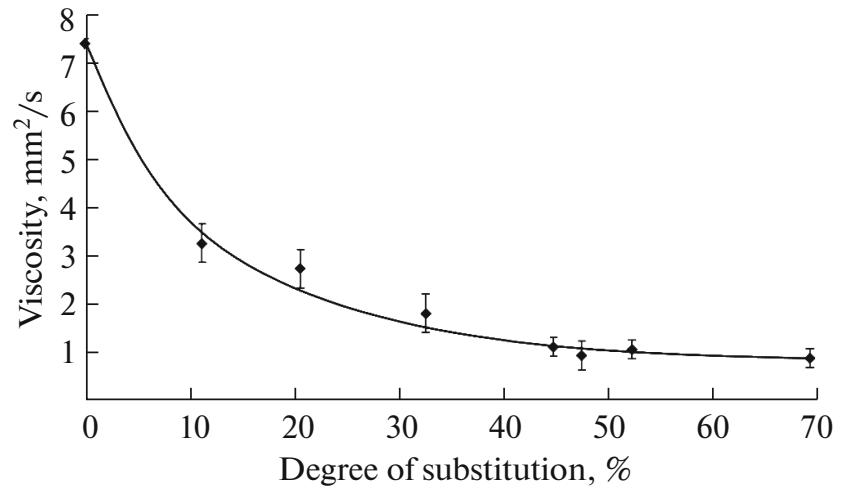

Fig. 4. Kinematic viscosity of aqueous mHA solutions $(0.5 \mathrm{mg} / \mathrm{mL})$ as a function of the degree of substitution. Data shown are the averages of three parallel measurements.

Savelyev et al. [15]. In preparing PCCs, flavin mononucleotide (also known as vitamin B2), which, like $\mathrm{HA}$, is a biocompatible endogenous component of the composition, was used as the photoinitiator. The PCCs were also supplemented with poly(ethylene glycol) diacrylate (PEGDA), which enabled us to fine tune the properties of scaffold, i.e., to decrease swelling and deformation of fabricated scaffolds after their contact with aqueous solutions, in particular, with a cell culture medium [17]. We note that, when using HA with DS $>60 \%$, the swelling coefficient decreases considerably and scaffolds can be prepared without PEGDA. We printed seven-layer scaffolds in the form of latticework with a period of $1.9 \mathrm{~mm}$ (Fig. 5).

The fabricated latticed scaffolds were incubated with a culture of human $\mathrm{Bj}-5$ ta fibroblasts for 14 days. MTT assays were performed for some samples on the 7th and 14th days. The assay showed not only the absence of cytotoxicity but also an increase in the number of cells over time (Fig. 6).

As can be seen in micrographs shown in Fig. 7, cells attached to the scaffolds unevenly, often in the form of cell aggregates at lattice nodes, which is in line with findings where cells displayed weak attachment to unmodified HA [28, 29]. In 14 days, however, the cells were capable of colonizing not only nodes but also lattice connections of the scaffolds.

The microscopy findings and MTT assay indicate the absence of cytotoxicity, moderate adhesion of cells to the scaffold surface, and the presence of cell growth during a 14-day incubation period. Adhesion of cells to the scaffold surface can be improved by coating it with other biocompatible materials that form a polyelectrolyte complex with HA, e.g., collagen, chitosan, or poly-L-lysine [30].

\section{EXPERIMENTAL}

\section{Materials}

Sodium salt of hyaluronic acid (HA; $100 \mathrm{kDa}$ ), glycidyl methacrylate (GMA), triethylamine (TEA), tetraethylammonium bromide (TEAB), potassium permanganate $\left(\mathrm{KMnO}_{4}\right)$, and poly(ethylene glycol) diacrylate (PEGDA; 575 Da) were obtained from Merck (Germany); and acetone, dimethylformamide (DMF), dimethyl sulfoxide, and concentrated hydrochloric acid were obtained from Khimmed (Russia). All the reagents were used as received. Flavin mononucleotide (Farmstandart, Russia) and amphotericin B (OAO Sintez, Russia) used in this work were obtained commercially. Penicillin-streptomycin, phosphate-buffered saline ( $\mathrm{pH}$ 7.4), and DMEM medium containing fetal bovine serum at $10 \%$ and

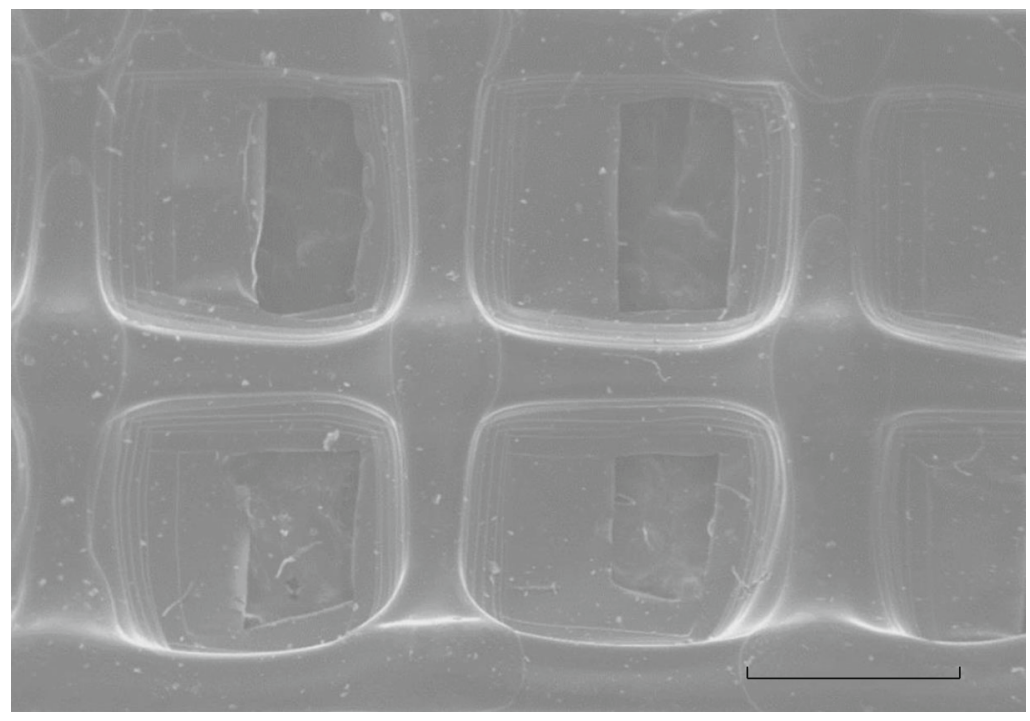

Fig. 5. Scanning electron microscopy image of a mHA-based scaffold. Scale bar: $1 \mathrm{~mm}$. 


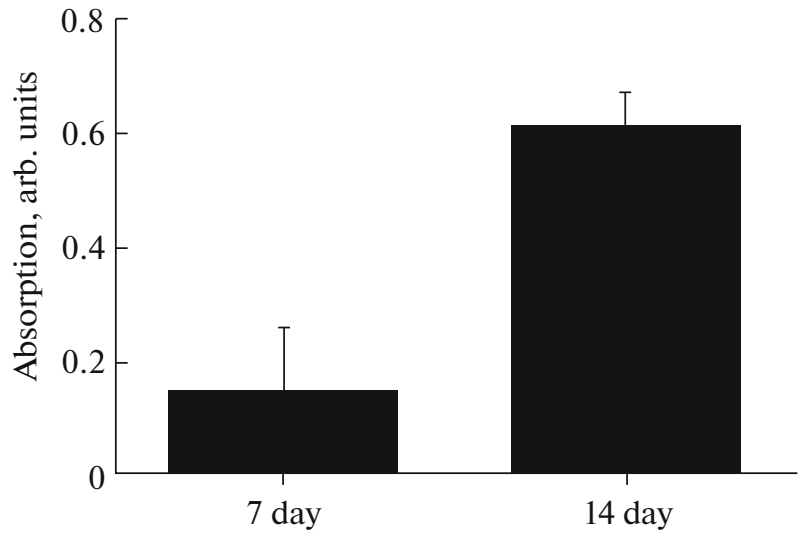

Fig. 6. Growth of immortalized human Bj-5ta fibroblasts on the scaffold surface and the results of MTT assay (the data are averages of five parallel measurements).

3-(4,5-dimethylthiazol-2-yl)-2,5-diphenyltetrazolium bromide (MTT), a dye, were acquired from PanEco (Russia).

\section{Preparing $m H A$}

To carry out the reaction, HA was dissolved in distilled water to make a concentration of 0.50 or $0.77 \mathrm{wt} \%$.
After the dissolution of HA was complete, DMF was added so that the volume ratio of water to DMF was $1.6: 1$ (or $1.2: 1$ ) in order to have a mixed aqueous organic solvent for dissolution of GMA. To control microorganism growth, the reaction mixture was supplemented with $500 \mu \mathrm{L}$ of penicillin-streptomycin $(5000 \mathrm{U} / \mathrm{mL}$ of penicillin $\mathrm{G}$ and $5000 \mu \mathrm{g} / \mathrm{mL}$ of streptomycin) and $128 \mu \mathrm{L}$ of amphotericin $\mathrm{B}(5 \mathrm{mg} / \mathrm{mL})$ per $100 \mathrm{~mL}$ of water. To investigate the effect of phase transfer catalysts, TEA or TEAB were added to the reaction mixture so that the weight ratio of $\mathrm{HA}$ to a catalyst was $1: 4.2$ or $1: 0.64$, respectively. After all the components were thoroughly mixed, GMA was added to obtain a number of different HA : GMA ratios $(\mathrm{g} / \mathrm{mL}): 1: 6,1: 8,1: 12,1: 14,1: 18$, and $1: 24$. After the GMA dissolved, the reaction mixture was held at either 25 or $40^{\circ} \mathrm{C}$ for $1,2,3$, or 4 days under continuous stirring. To cease the reaction and isolate mHA, the target product was precipitated by adding a sevenfold excess of acetone, and the resulting precipitate was separated by centrifugation. The product was purified by dissolving the precipitate in distilled water and subjecting it to dialysis against a tenfold excess of distilled water for three days, with the water being changed on a daily basis. The purified product was freeze-dried for two days, i.e., until the water was completely removed.
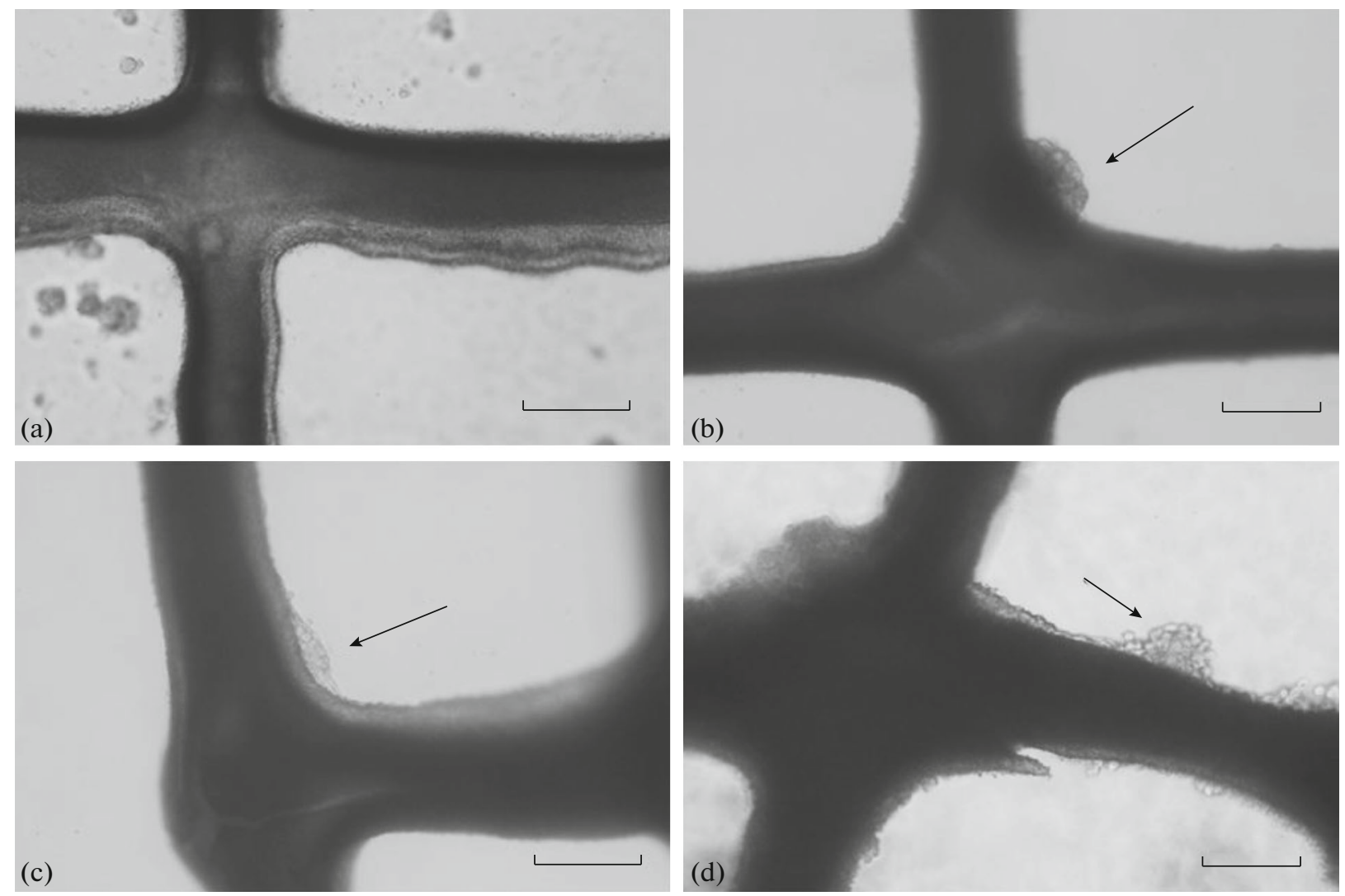

Fig. 7. Optical microscopy image of scaffolds with a culture of human Bj-5ta fibroblasts incubated for (a) 1, (b) 6, (c) 9 , and (d) 14 days. Cell aggregates are indicated with arrows. Scale bar: $300 \mu \mathrm{m}$. 


\section{Determining the Degree of Substitution (DS) of $m H A$ by the Wagner Reaction}

Quantitative determination of grafted vinyl groups was based on the reaction of $\mathrm{KMnO}_{4}$ and double bonds in mHA, which was followed by observing a color change. For this, $1 \mathrm{~mL}$ of a standard aqueous $\mathrm{KMnO}_{4}$ solution $(0.025 \%)$ was titrated with an aqueous $0.5 \%$ mHA solution until a complete color change occurred (the color changed from violet to yellow). The completion of reaction was established spectrophotometrically by observing the disappearance of absorption peaks related to $\mathrm{KMnO}_{4}$ in the region of 450-600 nm. All absorption spectra were registered on an Evolution $201 \mathrm{UV}-$ Vis spectrophotometer (Thermo Fisher Scientific, United States).

The degree of substitution (DS) of HA with vinyl groups was defined as the ratio between the quantity of grafted repeat units with double bonds to the total quantity of disaccharide units in a considered sample. The amount of double bonds that was required to ensure complete reduction of the standard $\mathrm{KMnO}_{4}$ solution was determined by titrating this standard with a solution of (unbound) GMA, as described in Sochilina et al. [24]. The quantity of disaccharide units was determined from the quantity of the analyte mHA that was required for complete reduction of the standard $\mathrm{KMnO}_{4}$ solution.

\section{Measuring the Viscosity of $m H A$ Solutions}

The kinematic viscosity of diluted $\mathrm{mHA}$ solutions (0.5\%) was measured using VPZh-2 0.34 and VPZh-2 0.56 viscometers (OOO EKROSKHIM, Russia). In measuring viscosity, the time period required for a liquid to flow out of the viscometer bulb at $37^{\circ} \mathrm{C}$ was measured, and the viscosity was calculated by the formula:

$$
V=\frac{g}{9.807} \times K \times T
$$

where $V$ is the kinematic viscosity of liquid, $\mathrm{mm}^{2} / \mathrm{s} ; g$ is the acceleration of gravity at the measurement location, $\mathrm{m} / \mathrm{s}^{2} ; K$ is the viscometer constant, $\mathrm{mm}^{2} / \mathrm{s}^{2}$; and $T$ is the time required for the liquid to flow out, $\mathrm{s}$. The viscometer constants were $0.003410 \mathrm{~mm}^{2} / \mathrm{s}^{2}$ for VPZh-2 0.34 and $0.008745 \mathrm{~mm}^{2} / \mathrm{s}^{2}$ for VPZh-2 0.56 . Because the viscosity of mHA samples varies in a broad range, we used the VPZh-2 0.34 viscometer for samples with DS $>45 \%$, and the VPZh-2 0.56 viscometer was used for samples with DS $<45 \%$ and unmodified HA.

\section{Preparing $\mathrm{mHA}$-Based Photocurable Compositions (PCCs) and Scaffolding Materials}

Photocurable compositions were prepared by combining mHA, PEGDA, and flavin mononucleotide in phosphate-buffered saline and subjecting to ultrason- ication for $1 \mathrm{~h}$ in an ultrasonic bath. The mixture was then left in the dark for $24 \mathrm{~h}$ and subjected to ultrasonication for $1 \mathrm{~h}$ again in order to obtain homogeneous PCCs. These steps were repeated if necessary, i.e., in cases when homogeneous compositions were not formed at the first attempt. The final proportions of the components were: $20 \mathrm{wt} \%$ of mHA, $5 \mathrm{wt} \%$ of PEGDA, and 0.01 wt $\%$ of flavin mononucleotide, which is the same as in a study by Savelyev et al. [15]. To prevent premature photocuring, the PCCs were handled only under yellow light.

Scaffolds were fabricated in the form of latticed structures by 3D printing. First, a syringe with a die hole in the form of capillary with a length of $5 \mathrm{~mm}$ and a diameter of $250 \mu \mathrm{m}$ was charged with a PCC. The PCC was extruded by pressing the syringe's plunger. The rate of plunger displacement and the $X-Y-Z$ positions of the syringe were controlled by the $\mathrm{G}$ code within the Repetier software. The extruded PCC was photocured by exposure to blue radiation $(450 \mathrm{~nm}$, intensity of $70 \mathrm{~mW} / \mathrm{cm}^{2}$ ) from a laser. To ensure that crosslinking was fully complete, the fabricated scaffolds were additionally held in a sealed container where thery were exposed to laser radiation with a wavelength of $450 \mathrm{~nm}$ and an intensity of $5 \mathrm{~mW} / \mathrm{cm}^{2}$ for $3 \mathrm{~h}$.

\section{In vitro Testing of $m H A$ Scaffolds with Cells}

mHA scaffolds were placed on a nonadhesive agarose-coated 24-well plate, and $10^{5}$ cells of the immortalized human fibroblast $\mathrm{Bj}-5$ ta cell line contained in $1 \mathrm{~mL}$ of DMEM medium with $10 \%$ of fetal bovine serum were added to each well (the cells were obtained from the Tumor Strain Bank, N.N. Blokhin Russian Cancer Research Center, Ministry of Health, Russia). The cells were incubated at $37^{\circ} \mathrm{C}$ and $5 \% \mathrm{CO}_{2}$ for 14 days, and the medium was fully refreshed every third day. Observations of cell attachment and development were made using an Olympus CKX35 inverted microscope (Olympus, Japan).

\section{MTT Assay}

The cell growth on the scaffold surface and investigation of the dynamics of matrix colonization were quantitatively evaluated by MTT assay. For this, the MTT dye was added to wells of a 24-well plate containing scaffolds with attached cells to a final concentration of $0.5 \mathrm{mg} / \mathrm{mL}$ followed by incubation at $37^{\circ} \mathrm{C}$ and $5 \% \mathrm{CO}_{2}$ for $3 \mathrm{~h}$. The scaffolds with dyed cells were then transferred in clean wells and washed with phosphate-buffered saline to remove unattached cells, and the formed formazan product was eluted with dimethyl sulfoxide ( $400 \mu \mathrm{L}$ per well, $20 \mathrm{~min}$ at room temperature). The formazan concentration was determined spectrophotometrically at $570 \mathrm{~nm}$ using a Mul- 
tiskan FC photometer (United States). Scaffolds that were not populated with cells were used as controls.

\section{CONCLUSIONS}

In this work, we proposed an approach to effectively control the degree of substitution (DS) of glycidyl methacrylate (GMA)-modified hyaluronic acid (HA) by varying reaction parameters $(\mathrm{pH}$, temperature, catalyst type, and the HA/GMA ratio). This approach enables precise control over the properties of HA derivatives, depending on the end goal for their use. As a result of this study, we prepared photocurable composites optimised for $3 \mathrm{D}$ printing and fabricated scaffolds that can ensure adhesion of cells to the surface, as well as their growth and development.

This study is the basis for further development of a technology for fabrication of HA-based scaffolds, including their in situ formation in the living body. In addition, the approach developed here enables us to fabricate scaffolds with unique architecture, e.g., in the form of hollow cylinders to replace damaged vessels, which opens new possibilities for regenerative medicine.

\section{ACNOWLEDGMENTS}

We are grateful to D.A. Khochenkov for providing us with immortalized human $\mathrm{Bj}-5$ ta fibroblasts.

\section{FUNDING}

The work was supported part by the Russian Foundation for Basic Research (project no. 19-33-90285-doctoral students; modification of hyaluronic acid and studies of its properties) and part by the Ministry of Science and Higher Education of the Russian Federation within the State assignment Federal Scientific Research Centre "Crystallography and Photonics" of Russian Academy of Science (photocuring of polymer compositions).

\section{COMPLIANCE WITH ETHICAL STANDARDS}

This article does not contain any studies involving animals or human participants performed by any of the authors.

\section{Conflict of Interests}

The authors declare that they have no conflict of interest.

\section{OPEN ACCESS}

This article is licensed under a Creative Commons Attribution 4.0 International License, which permits use, sharing, adaptation, distribution and reproduction in any medium or format, as long as you give appropriate credit to the original author(s) and the source, provide a link to the Creative Commons licence, and indicate if changes were made. The images or other third party material in this article are included in the article's Creative Commons licence, unless indicated otherwise in a credit line to the material. If material is not included in the article's Creative Commons licence and your intended use is not permitted by statutory regulation or exceeds the permitted use, you will need to obtain permission directly from the copyright holder. To view a copy of this licence, visit http://creativecommons.org/licenses/by/4.0/.

\section{REFERENCES}

1. Langer, R. and Vacanti, J., Science, 1993, vol. 260, pp. 920-926. https://doi.org/10.1126/science.8493529

2. Howard, D., Buttery, L.D., Shakesheff, K.M., and Roberts, S.J., J. Anat., 2008, vol. 213, pp. 66-72. https://doi.org/10.1111/j.1469-7580.2008.00878.x

3. Almouemen, N., Kelly, H.M., and O'Leary, C., Comput. Struct. Biotechnol. J., 2019, vol. 17, pp. 591-598. https://doi.org/10.1016/j.csbj.2019.04.008

4. Collins, M.N. and Birkinshaw, C., Carbohydr. Res., 2013, vol. 92, pp. 1262-1279. https://doi.org/10.1016/j.carbpol.2012.10.028

5. Nikolova, M.P. and Chavali, M.S., Bioact. Mater., 2019, vol. 4, pp. 271-292. https://doi.org/10.1016/j.bioactmat.2019.10.005

6. Qu, H., Fu, H., Han, Z., and Sun, Y., RSC Adv., 2019, vol. 9, pp. 26252-26262. https://doi.org/10.1039/C9RA05214C

7. Khvostov, M.V., Tolstikova, T.G., Borisov, S.A., and Dushkin, A.V., Russ. J. Bioorg. Chem., 2019, vol. 45, pp. 438-450. https://doi.org/10.1134/S1068162019060219

8. Kim, H., Jeong, H., Han, S., Beack, S., Hwang, B.W., Shin, M., Oh, S.S., and Hahn, S.K., Biomaterials, 2017, vol. 123, pp. 155-171.

https://doi.org/10.1016/j.biomaterials.2017.01.029

9. Mohammadi, F., Samani, S.M., Tanideh, N., and Ahmadi, F., Adv. Pharm. Bull., 2018, vol. 8, pp. 11-19. https://doi.org/10.15171/apb.2018.002

10. Guan, Y., You, H., Cai, J., Zhang, Q., Yan, S., and You, R., Carbohydr. Res., 2020, vol. 239, p. 116232. https://doi.org/10.1016/j.carbpol.2020.116232

11. Fujita, S., Hara, S., Hosono, A., Sugihara, S., Uematsu, H., and Suye, S., Adv. Polym. Technol., 2020, vol. 2020, pp. 1-7. https://doi.org/10.1155/2020/1470819

12. Fahmy, A., Kamoun, E.A., El-Eisawy, R., ElFakharany, E.M., Taha, T.H., El-Damhougy, B.K., and Abdelhai, F., J. Braz. Chem. Soc., 2015, vol. 26, pp. $1466-1474$. https://doi.org/10.5935/0103-5053.20150115

13. Khunmanee, S., Jeong, Y., and Park, H., J. Tissue Eng., 2017, vol. 8, p. 204173141772646. https://doi.org/10.1177/2041731417726464

14. Poldervaart, M.T., Goversen, B., de Ruijter, M., Abbadessa, A., Melchels, F.P.W., Öner, F.C., Dhert, W.J.A., Vermonden, T., and Alblas, J., PLoS One, 2017, vol. 12, e0177628.

https://doi.org/10.1371/journal.pone.0177628 
15. Savelyev, A.G., Sochilina, A.V., Akasov, R.A., Mironov, A.V., Semchishen, V.A., Generalova, A.N., Khaydukov, E.V., and Popov, V.K., Sovrem. Tehnol. Med., 2018, vol. 10, p. 88. https://doi.org/10.17691/stm2018.10.1.11

16. Lam, T., Dehne, T., Kruger, J.P., Hondke, S., Endres, M., and Thomas, A., J. Biomed. Mater. Res. Part B Appl. Biomater., 2019, vol. 107, pp. 2649-2657. https://doi.org/10.1002/jbm.b.34354

17. Savelyev, A.G., Bardakova, K.N., Khaydukov, E.V., Generalova, A.N., Popov, V.K., Chichkov, B.N., and Semchishen, V.A., J. Photochem. Photobiol. A Chem., 2017, vol. 341, pp. 108-114. https://doi.org/10.1016/j.jphotochem.2017.03.026

18. Guo, C., Qu, X., Rangaswamy, N., Leehy, B., Xiang, C., and Rice, D., PLoS One, 2018, vol. 13, e0196529. https://doi.org/10.1371/journal.pone.0196529

19. Baier, L.J., Bivens, K.A., Patrick, C.W., Jr., and Schmidt, C.E., Biotechnol. Bioeng., 2003, vol. 82, pp. 578-589. https://doi.org/10.1002/bit.10605

20. Oudshoorn, M.H.M., Rissmann, R., Bouwstra, J.A., and Hennink, W.E., Polymer, 2007, vol. 48, pp. 19151920. https://doi.org/10.1016/j.polymer.2007.01.068

21. Bencherif, S.A., Srinivasan, A., Horkay, F., Hollinger, J.O., Matyjaszewski, K., and Washburn, N.R., Biomaterials, 2008, vol. 29, pp. 1739-1749. https://doi.org/10.1016/j.biomaterials.2007.11.047

22. Tsanaktsidou, E., Kammona, O., and Kiparissides, C., Eur. Polym. J., 2019, vol. 114, pp. 47-56. https://doi.org/10.1016/j.eurpolymj.2019.02.024
23. Yousefi, F., Kandel, S., and Pleshko, N., Appl. Spectrosc., 2018, vol. 72, pp. 1455-1466. https://doi.org/10.1177/0003702818785353

24. Sochilina, A.V., Savelyev, A.G., Demina, P.A., Sizova, S.V., Zubov, V.P., Khaydukov, E.V., and Generalova, A.N., Meas. Sci. Technol., 2019, vol. 30, p. 075102. https://doi.org/10.1088/1361-6501/ab0fb4

25. Reis, A.V., Fajardo, A.R., Schuquel, I.T.A., Guilherme, M.R., Vidotti, G.J., Rubira, A.F., and Muniz, E.C., Org. Chem., 2009, vol. 74, pp. 3750-3757. https://doi.org/10.1021/jo900033c

26. Li, Q., Wang, D., and Elisseeff, J.H., Macromolecules, 2003, vol. 36, pp. 2556-2562. https://doi.org/10.1021/ma021190w

27. Lowry, K.M. and Beavers, E.M., J. Biomed. Mater. Res., 1994, vol. 28, pp. 1239-1244. https://doi.org/10.1002/jbm.820281014

28. Pavesio, A., Renier, D., Cassinelli, C., and Morra, M., Med. Device Technol., 1997, vol. 8, no. 7, pp. 2021, 24-27.

29. Morra, M. and Cassineli, C., J. Biomater. Sci. Polym. Ed., 1999, vol. 10, pp. 1107-1124. https://doi.org/10.1163/156856299X00711

30. Yamanlar, S., Sant, S., Boudou, T., Picart, C., and Khademhosseini, A., Biomaterials, 2011, vol. 32, pp. 5590-5599.

https://doi.org/10.1016/j.biomaterials.2011.04.030

Translated by A. Kukharuk 